\title{
Aplicación de un ciclo de mejora de la docencia en la asignatura producción y empresa audiovisual II del grado en comunicación audiovisual
}

\section{Application of a Cycle of Teaching Improvement in the Subject Production and Audiovisual Enterprise II in the Audiovisual Media Degree}

Miguel Ángel Pérez Gómez

ORCID: https://orcid.org/0000-0002-7315-1809

Universidad de Sevilla

Dpto. de Comunicación audiovisual y Publicidad mperez21@us.es

Fecha de recepción:

Fecha de aceptación:

DOI: http://dx.doi.org/10.12795/9788447221912.109

Pp.: 2435-2451 


\section{Resumen}

En la presente comunicación se lleva a cabo un análisis pormenorizado de los resultados del Ciclo de Mejora Docente implementado en la asignatura Producción y empresa audiovisual II, de 3o del Grado en comunicación audiovisual durante el primer cuatrimestre del curso 2019/2020. Se trata de un CMD de ocho horas de duración, cuatro sesiones de dos horas, aplicada a una asignatura que tiene como proyecto general la realización de un cortometraje. En este texto se explica de manera detallada los principios didácticos sintetizados en el Programa de Formación Docente CGDU 4.

Palabras clave: Producción y empresa audiovisual II, Comunicación audiovisual, Producción cinematográfica, Docencia universitaria, Experimentación docente universitaria.

\section{Abstract}

In this paper, is carried out a detailed analysis of the results of the Teaching Improvement Cycle implemented in the subject Production and Media Enterprise II, in audiovisual media degree during the first four months of the 2019/2020 academic year. This is an eight-hour CMD, four sessions of two hours each, applied to a subject whose general project is to make a short film. It explains the didactic principles synthesized in the CGDU 4 Teacher Training Program.

Keywords: Production and Media Enterprise II, Audiovisual Media, Film Production, University teaching, University teaching experimentation.

Jornadas de Formación e Innovación Docente del Profesorado | № 2 (2019) Esta obra se distribuye con la licencia Creative Commons 


\section{Descripción del contexto de la intervención}

La asignatura Producción y Empresa audiovisual II se imparte en el primer cuatrimestre del tercer curso del Grado en Comunicación Audiovisual y del Doble Grado en Periodismo y Comunicación Audiovisual. Es una asignatura de carácter obligatorio que tiene como particularidad que para superarla los alumnos deben de realizar un examen y un ejercicio práctico consistente en producir un cortometraje. A nivel académico, el cortometraje sirve como trabajo único para: la asignatura de producción, anteriormente citada, Realización III, desde la cual se evalúan aspectos como la realización y posproducción del cortometraje; y Puesta en escena desde la cual se hace el diseño visual de esta pieza audiovisual.

\section{Diseño previo del Ciclo de Mejora Docente}

Este Ciclo de Mejora Docente viene integrado en un proyecto que pretende enfrentar a los alumnos a un gran problema: la realización de un cortometraje. Esto nos lleva a proponer un programa que ponga al alumno frente a una serie de problemáticas que se asemejan a situaciones profesionales reales, tal como proponen Dolmans et al (2005). La asignatura en sí misma refuerza algunos conceptos y aspectos prácticos estructurales, que permiten mostrar posibles salidas profesionales para el alumnado.

\section{Mapa de contenidos}

Como la aplicación del CMD ha sido con el proyecto audiovisual ya iniciado, se ha utilizado para dar relevancia a cada uno de los roles dentro de cada uno de los grupos. Pero también para cerrar el apartado de preproducción 
del cortometraje, subrayando la importancia de la planificación previa a la grabación. Para ello se ha orientado la acción docente a determinar tres áreas de trabajo para esta actividad de mejora: construcción de un casting, acotación de aspectos legales y de contratación, y para finalizar, la realización de un plan de rodaje; en el cual confluyen el trabajo realizado previamente por parte de los estudiantes.

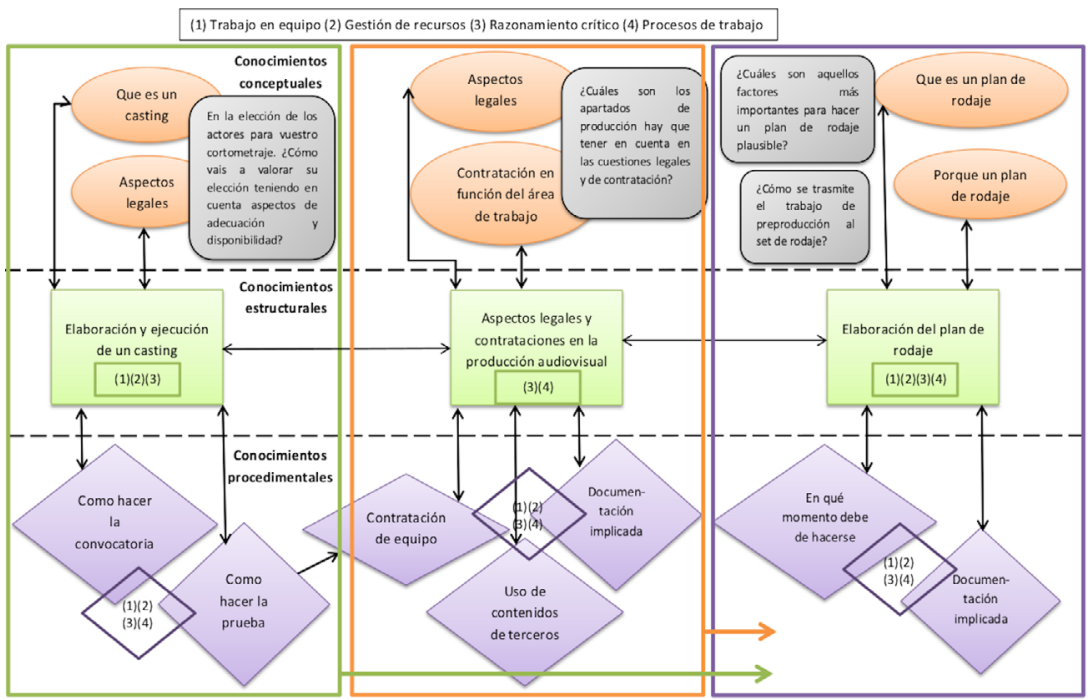

Figura 1. Mapa de contenidos para Producción y empresa audiovisual II

Los elementos que han orientado la actividad docente son: la determinación de los objetivos didácticos y la elaboración de un mapa de contenidos sustentado por cuatro grandes problemas que estructuran cada una de las sesiones de trabajo. El mapa de contenidos constituye una herramienta fundamental para entender la relación entre los diferentes contenidos y la construcción de unos problemas que realmente sean útiles para el alumnado. Sin apartarlos del ejercicio principal. Pretendiendo "crear un entorno para el aprendizaje crítico natural" (Bain, 2007). Los bloques temáticos contenidos en el Proyecto Docente de la asignatura se han resumido en el mapa conceptual mostrado. 
Modelo metodológico posible

En el modelo metodológico diseñado, el estudiante será capaz de resolver los cuatro problemas planteados. Esta concepción metodológica implica, por un lado, el compromiso del alumno en el desarrollo de cada una de las tareas. No hay una solución para todos los proyectos, sino que cada grupo de trabajo debe de encontrar la suya. Incluso cada alumno, de manera individual y dependiendo del rol que tenga dentro del equipo, deberá encontrar su propia solución. Para la realización de este modelo se han adoptado las pautas sobre la importancia del trabajo en grupo de Finkel (2008, p. 153-170).

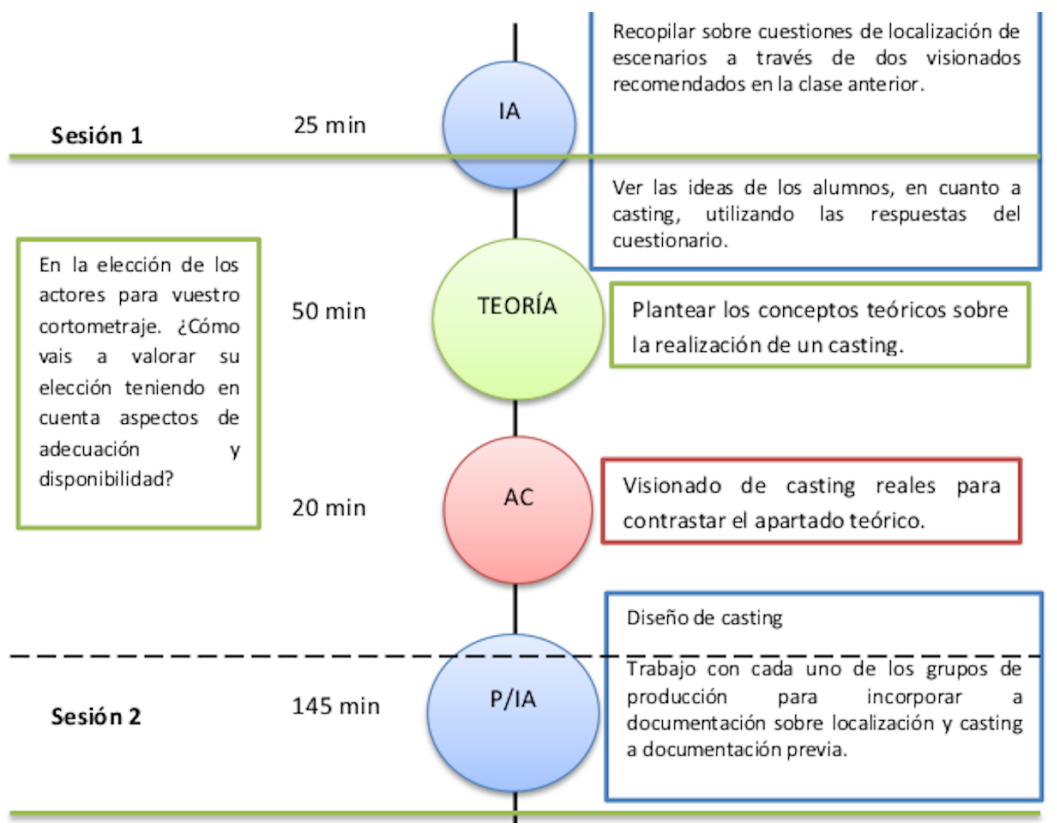

Figura 2. Modelo metodológico posible para las sesiones 1 y 2

Jornadas de Formación e Innovación Docente del Profesorado | № 2 (2019) Esta obra se distribuye con la licencia Creative Commons Reconocimiento-NoComercial-SinObraDerivada 4.0 Internacional (CC BY-NC-ND 4.0.) 


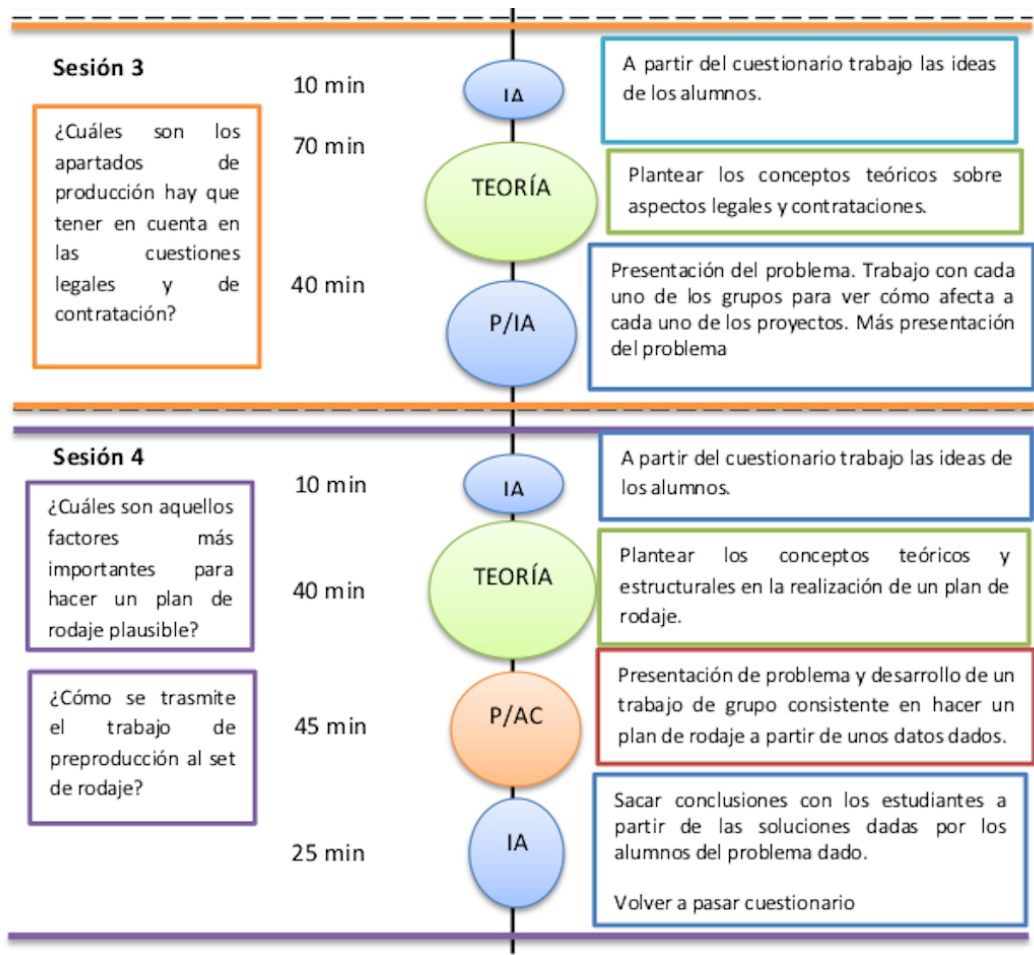

Figura 3. Modelo metodológico posible para las sesiones 3 y 4

Dentro de las diferentes sesiones se han realizado una serie de actividades destinadas a reforzar el aprendizaje del alumnado. A continuación pueden verse las fichas de las mismas.

Tabla 1. Secuencia de actividades del ciclo de mejora

Sesión 1: Introducción a la realización de castings

\begin{tabular}{l|l|l|l}
\hline №1.Ideas de los alumnos & Ver las conclusiones de los alumnos & Tiempo 15'
\end{tabular} tras los visionados propuestos en la clase anterior.

Inicio esta sesión recopilando las conclusiones que los alumnos han extraído de los visionados de Los cronocrímenes (Nacho Vigalondo, 2007) y ¿Quién puede matar a un niño? (Narciso Ibáñez Serrador, 1976), sobre cuestiones de localización. Es importante enlazar con la clase anterior ya que al final del CIMA los estudiantes tienen que volver a trabajar este tema.

Recursos: Visionado de películas, intervención del profesor y los alumnos. 
Problema 1: En la elección de los actores para vuestro cortometraje. ¿Cómo vais a valorar su elección teniendo en cuenta aspectos de adecuación y disponibilidad?

\begin{tabular}{|l|l|l}
\hline No2. Ideas de los alumnos & $\begin{array}{l}\text { Trabajar con las respuestas de los } \\
\text { alumnos al cuestionario }\end{array}$ & Tiempo 10' \\
\hline
\end{tabular}

Se recopilan las ideas de los cuestionarios de los alumnos en la formulación de la primera pregunta, para extraer unas conclusiones previas antes de impartir el bloque teórico.

Recursos: Intervención de los alumnos y el profesor

\begin{tabular}{|l|l|l}
\hline № 3 & Teoría & Tiempo 50
\end{tabular}

En este bloque se profundiza en cuestiones teóricas y prácticas sobre la realización de castings con el fin de que puedan: crear perfiles de actores a partir de los personajes del guion, diseño de un casting y puesta en práctica.

Recursos: Powerpoint, intervención del profesor y los alumnos.

\begin{tabular}{l|l|l}
\hline $\begin{array}{l}\text { No-4.Actividad de } \\
\text { contraste }\end{array}$ & Vídeo & Tiempo 20' \\
\hline
\end{tabular}

La proyección de un vídeo en este punto de la clase sirve para que los alumnos contrasten sus conocimientos personales y los teóricos vistos anteriormente con el vídeo propuesto que muestra como son los castings profesionales. Las piezas audiovisuales en cuestión es How to Hold an Audition (https://www.youtube.com/ watch?V=4PoCjSF-QBU) sobre la técnica a llevar a cabo en el desarrollo de un casting.

Recursos: Visionado de vídeos, intervención del profesor y los alumnos.

\begin{tabular}{l|l|l}
\hline No5. Ideas de los & Presentación problema y problema & 25
\end{tabular}

alumnos.

Una vez vistas las cuestiones teóricas y prácticas sobre el casting se les plantea a los alumnos un ejercicio a tres bandas: la elaboración de un perfil actoral a partir del guion, la creación de una convocatoria y la de un protocolo de casting. Se presenta el problema dando indicaciones sobre el trabajo a abordar y luego voy de grupo en grupo recogiendo las dudas planteadas. El ejercicio se cierra en la segunda sesión compilando las conclusiones de los alumnos.

Recursos: Problema, intervención del profesor y trabajo de grupo.

Sesión 2: Aplicar los últimos avances en la producción en documentos previos

\begin{tabular}{|l|l|l|}
\hline $\begin{array}{l}\text { No1. Ideas de los } \\
\text { alumnos. }\end{array}$ & $\begin{array}{l}\text { Ver las conclusiones de los alumnos } \\
\text { tras el ejercicio propuesto en la } \\
\text { clase anterior. }\end{array}$ & Tiempo 20' \\
\hline
\end{tabular}

Tras la realización del ejercicio, que se inició en la clase anterior, se recogen las dudas principales, los problemas que ha generado dicho ejercicio y se anotan las conclusiones extraídas del ejercicio anterior para que puedan llevar a cabo el casting para la producción del cortometraje.

Recursos: Ideas de los alumnos e intervenciones del profesor.

\begin{tabular}{|l|l|l|}
\hline $\begin{array}{l}\text { No2. Ideas de los } \\
\text { alumnos. }\end{array}$ & $\begin{array}{l}\text { Revisión de cada uno de los } \\
\text { proyectos de producción }\end{array}$ & Tiempo 100' \\
\hline
\end{tabular}


En esta actividad se procede a revisar cómo se han aplicado los conocimientos en localizaciones y casting a documentos de producción previos, en la preproducción es habitual ir y volver a los documentos para hacer actualizaciones. Es conveniente cerrar esta fase revisando cada proyecto, ya que a partir de este momento se debe preparar toda la documentación para realizar un plan de rodaje.

Recursos: Ideas de los alumnos e intervenciones del profesor. Trabajo en grupo.

Sesión 3: Introducción a la contratación y aspectos legales relacionados con los cortometrajes.

Problema 2: ¿Cuáles son los apartados de producción hay que tener en cuenta en las cuestiones legales y de contratación?

\begin{tabular}{|l|l|l} 
№1. Ideas de los alumnos & Trabajar con las respuestas de los & Tiempo 10'
\end{tabular} alumnos al cuestionario

Se recopilan las ideas del cuestionario en la formulación de la segunda pregunta y se extraen unas conclusiones previas antes de impartir el bloque teórico.

Recursos: Intervención de los alumnos y el profesor

\begin{tabular}{l|l|l}
\hline №2 & Teoría & Tiempo 70'
\end{tabular}

En este bloque se profundiza en cuestiones teóricas y prácticas sobre los aspectos legales que se han de tener en cuenta en la contratación de profesionales y el uso de contenidos creados por terceros.

Recursos: Powerpoint, intervención del profesor y los alumnos.

\begin{tabular}{l|l|l|}
\hline №3 & Presentación problema y problema & Tiempo 40'
\end{tabular}

A partir de la división en el tipo de contratación en función del área de trabajo, presento un ejercicio práctico en clase en el que tienen desarrollar diferentes perfiles de contratación para su cortometraje. Teniendo en cuenta lo visto en fases previas del trabajo, así como de la fase de casting.

Recursos: Trabajo en grupo. Intervención del profesor y de los alumnos.

Sesión 4: Desarrollo de un plan de rodaje.

Problema 3: ¿Cuáles son aquellos factores más importantes para hacer un plan de rodaje plausible?

Problema 4: ¿Cómo se trasmite el trabajo de preproducción al set de rodaje?

\begin{tabular}{l|l|l} 
№1. Ideas de los & Trabajar con las respuestas de los & Tiempo 10'
\end{tabular} alumnos. $\quad$ alumnos al cuestionario

Se recopilan las ideas del cuestionario en la formulación de la tercera y cuarta pregunta y se extraen unas conclusiones previas antes de impartir el bloque teórico.

Recursos: Intervención de los alumnos y el profesor

\begin{tabular}{|l|l|l|}
\hline №2 & Teoría & Tiempo 40' \\
\hline Se exponen cuestiones fundamentales a la hora de hacer un plan de rodaje poniendo
\end{tabular}

Se exponen cuestiones fundamentales a la hora de hacer un plan de rodaje poniendo de relieve aquellos elementos que podemos considerar como pilares básicos de la planificación en la producción audiovisual. 


\begin{tabular}{|l|l|l|}
\hline \multicolumn{2}{|l|}{ Recursos: Powerpoint, intervención del profesor y los alumnos. } \\
\hline $\begin{array}{l}\text { No3. Actividad de } \\
\text { contraste. }\end{array}$ & $\begin{array}{l}\text { Presentación de problema y } \\
\text { problema }\end{array}$ & Tiempo 45' \\
\hline
\end{tabular}

Se presentan al alumno una serie de ejercicios sobre el desarrollo de un plan de rodaje a partir de un calendario dado y unas fechas de disponibilidad del equipo técnico, creativo y las necesidades técnicas. Este problema lo tienen que resolver entre los miembros del equipo de trabajo.

Recursos: Trabajo en grupo. Intervención del profesor y de los alumnos.

№4. Ideas de los

alumnos.

\begin{tabular}{|l|l}
\hline $\begin{array}{l}\text { Ver soluciones y conclusiones } \\
\text { de los alumnos en el problema } \\
\text { propuesto. }\end{array}$ & Tiempo 10' \\
\hline
\end{tabular}

Una vez finalizado el ejercicio se anotan las soluciones aportadas por los alumnos en la resolución del problema planteado en la actividad anterior. Se busca que el alumno encuentre las soluciones más efectivas y evitar conflictos de disponibilidad entre los profesionales en una producción audiovisual.

Recursos: Ideas de los alumnos e intervenciones del profesor. Trabajo en grupo.

\begin{tabular}{|l|l|l}
\hline №5. Ideas de los & Pasar cuestionario final & Tiempo 15'
\end{tabular}

alumnos.

Una vez finalizado el CIMA se vuelve a pasar el cuestionario para comprobar los avances realizados de los alumnos.

Recursos: Cuestionario y respuestas de los alumnos.

Rivero y Porlán (2017, pp. 73-91) apuntan que la herramienta fundamental para conocer los modelos mentales de los estudiantes es el cuestionario; para ello se desarrolla un cuestionario inicial, que nos permita explorar al alumnado, y uno final. El primero sirve para elaborar un mapa de contenidos y el segundo para evaluar nuestra acción docente. El desarrollo de la asignatura me permite hacer las preguntas directamente sobre el proyecto que están desarrollando los estudiantes. El cuestionario que han tenido que responder los alumnos es el siguiente:

1. En la elección de los actores para vuestro papel ¿Cómo vais a valorar su elección teniendo en cuenta aspectos de adecuación o disponibilidad?

2. Sabemos que uno de los retos en la fase de preproducción son las cuestiones legales y de contratación. ¿Sabrías decirme en que apartados de producción hay que tener en cuenta cuestiones legales y de contratación? 
3. ¿Cuándo llegue el momento de hacer el plan de rodaje. ¿Cuáles crees que son aquellos factores más importantes para hacer un plan de rodaje plausible?

4. Una vez llegado el momento del rodaje y la posproducción del cortometraje ¿Cómo crees que se transmite el trabajo de preproducción al set de rodaje?

\section{Aplicación del CIMA}

Las sesiones se desarrollaron siguiendo la secuencia de actividades. La primera sesión con un ejercicio colectivo, pero dividido en dos partes: en primer lugar se recopilan las ideas sobre localización de escenarios a partir de la recomendación de visionados de dos películas de ciencia ficción españolas, en las que la selección de localizaciones es muy efectiva. La segunda se desarrolla con las ideas de los alumnos sobre el casting de actores. Para ello se utilizan las respuestas, ya analizadas, de los alumnos al cuestionario inicial. Se apuntan en la pizarra los grupos de respuestas sobre el tema, y con los propios estudiantes se jerarquizan, desde las menos recomendables a las más adecuadas. En el siguiente bloque se expone la teoría, los alumnos preguntan por casos actuales. Complemento esa explicación teórica con un vídeo en los que se muestran las dinámicas que se llevan a cabo en un casting y se muestran castings reales.

Para finalizar la sesión, y ya vinculado con el proyecto, se supervisa a cada uno de los grupos en tres labores: la creación del cartel de la convocatoria de casting, el desarrollo de la rutina de la prueba y la ficha que han de tener preparada para cada uno de los candidatos que se presenten. Para los tres ejercicios se resuelven las dudas concretas para cada proyecto. En esta sesión finalizan gran parte del trabajo. Una vez analizados los tres ejercicios de la clase anterior, se utilizan los resultados en la segunda sesión para resolver las últimas dudas a través de una puesta en común del trabajo de todos los grupos. Esta segunda sesión está dedicada en su integridad a una revisión de cada uno de los proyectos, es un cierre de un 
apartado de la producción cinematográfica y se recogen cuestiones impartidas en las últimas sesiones. Con cada grupo se revisa si hay que actualizar algunos documentos realizados previamente y se finaliza cada uno de los apartados de la producción.

La tercera sesión se centra en que los alumnos aprendan la importancia de la contratación como parte de la producción. Pero, principalmente, que al finalizar la clase puedan leer, entender y crear documentos que son fundamentales para la contratación en producción. Para ello, al igual que en la primera sesión, se anota en la pizarra los modelos dados por los alumnos y al lado se dibuja una tabla con los diferentes tipos de contratación. Con sus ideas se van situando cada uno de los modelos en cada apartado de la parrilla. Al separar los diferentes modelos de contratación cada uno de los alumnos sabe que tipo de contratación tiene que gestionar, vinculada a su rol dentro del equipo. El segundo bloque de trabajo de esta sesión es el apartado teórico, este al ser más árido para los alumnos, con respecto a otros temas de la asignatura, se necesita más tiempo para aclarar dudas a medida que se imparte la teoría. El último apartado de esta clase finaliza con cada grupo iniciando su estrategia de contrataciones. Deben de revisar toda la documentación del dossier del cortometraje para: empezar a listar el tipo de contratos que deben de hacer junto con la cantidad de cada uno de ellos y de qué tipo de contrato se debe de ocupar cada uno de los miembros del equipo de producción.

En la última sesión los alumnos deben de conjugar todos los recursos que han ido compilando hasta ahora, por dicho motivo es la sesión con más actividades. Los dos problemas planteados, dentro del ejercicio general, pretenden hacer pensar a los alumnos cómo van a llevar al set de rodaje todo el trabajo que están haciendo sobre el papel. La segunda actividad, de carácter teórico, consiste mostrarles cuales son las prioridades para hacer un calendario de trabajo. Tras presentar el ejercicio cada grupo se pone a trabajar con toda la información recogida hasta 
el momento y traspasarla a un calendario. Aquí las dudas que surgen es como se deben de codificar todas las informaciones en las plantillas dadas. Un plan de rodaje no se finaliza en una tarde pero dejo todos los conceptos desarrollados y todas las dudas resueltas en el tiempo que los alumnos tienen para mostrar sus ideas. La sesión finaliza con los alumnos respondiendo el cuestionario final. A partir ambos cuestionarios se han realizado cuatro escaleras de aprendizaje que muestran la evolución de las cuatro sesiones.

1. En la elección de los actores para vuestro papel ¿Cómo vais a valorar su elección teniendo en cuenta aspectos de adecuación o disponibilidad?

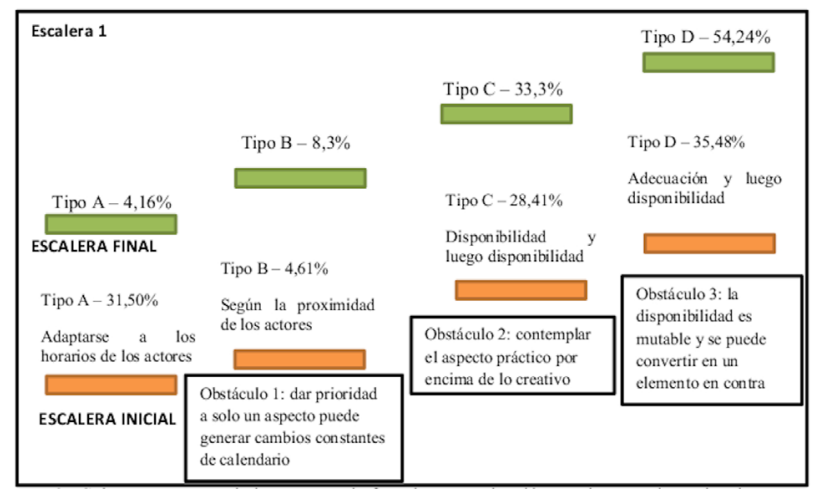

Figura 4. Escalera de aprendizaje correspondiente al primer problema

2. ¿Sabrías decirme en que apartados de producción hay que tener en cuenta cuestiones legales y de contratación?

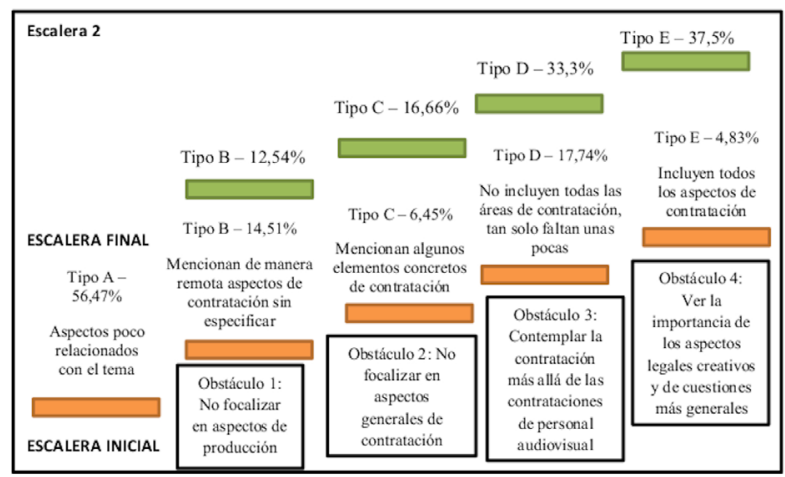

Figura 5. Escalera de aprendizaje correspondiente al segundo problema

Jornadas de Formación e Innovación Docente del Profesorado | № 2 (2019) Esta obra se distribuye con la licencia Creative Commons Reconocimiento-NoComercial-SinObraDerivada Internacional (CC BY-NC-ND 4.0.) 
3. Cuándo llegue el momento de hacer el plan de rodaje. ¿Cuáles crees que son aquellos factores más importantes para hacer un plan de rodaje plausible?

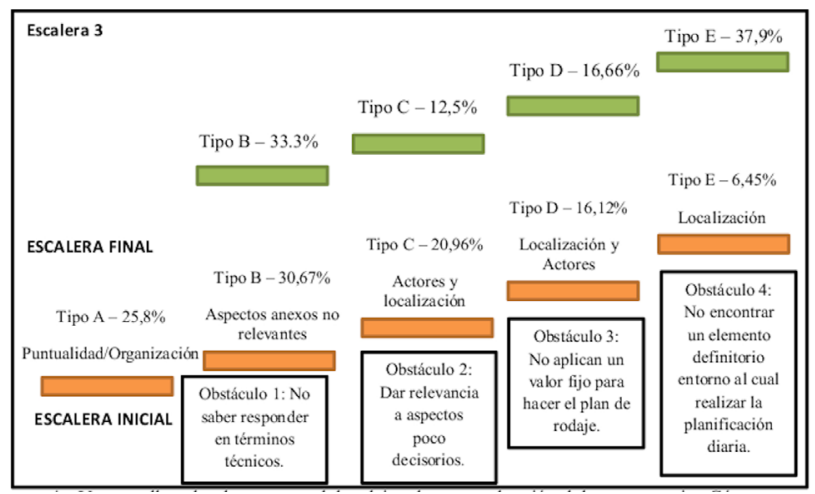

Figura 6. Escalera de aprendizaje correspondiente al tercer problema

4. Una vez llegado el momento del rodaje y la posproducción del cortometraje ¿Cómo crees que se transmite el trabajo de preproducción al set de rodaje?

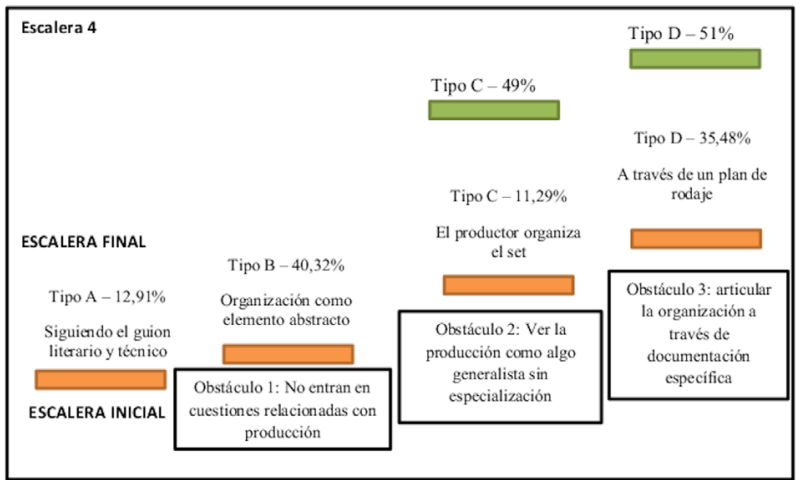

Figura 7. Escalera de aprendizaje correspondiente al cuarto problema

Analizando las escaleras de aprendizaje podemos observar lo siguiente:

En la primera pregunta se puede apreciar como los alumnos han pasado de una opción de respuesta puramente pragmática, a una en la que la adecuación de los actores y la disponibilidad de los mismos son dos factores 
con los que los estudiantes, en su faceta de productores, deben negociar para encontrar al actor que más les convenza. En el cuestionario final casi todo el alumnado se sitúa en los dos escalones más altos. La segunda pregunta, dedicada a la contratación y los aspectos legales, tiene una evolución más encadenada. Si bien en el cuestionario inicial más de la mitad de la clase se sitúa en el peldaño más bajo, en el final vemos como ese peldaño ha sido suprimido y que dos tercios del alumnado están en los dos peldaños más altos.

En el tercer problema se aprecia como hay un incremento en el escalón más alto. Sin embargo, a pesar de que en el cuestionario final se ha eliminado el escalón más bajo, un tercio de los alumnos tienen problemas para comprender los ejes de ordenación de un plan de rodaje. La última escalera es la que nos permite ver una evolución más notable. En la encuesta inicial el porcentaje que ocupan los dos escalones inferiores y los dos superiores es prácticamente igual. En la escalera final se puede apreciar que los alumnos pasan a estar en los dos escalones finales en una proporción similar.

También se ha realizado un cuadro de evolución de los estudiantes en la que se muestra el avance de un grupo de alumnos. Debido a la gran cantidad de estudiantes, unos 80 , se ha decidido tomar una muestra de diez pares de cuestionarios.

Tabla 2. Cuadro de evolución por estudiantes (muestra de alumnos)

\begin{tabular}{|l|c|c|c|c|c|c|c|c|c|c|c|c|c|}
\hline \multicolumn{10}{|c|}{ Niveles de desarrollo iniciales y finales } \\
\hline & \multicolumn{1}{|c|}{ Pregunta 1 } & \multicolumn{3}{|c|}{ Pregunta 2 } & \multicolumn{3}{|c|}{ Pregunta 3 } & \multicolumn{3}{|c|}{ Pregunta 4 } \\
\hline Sujeto 1 & $\mathrm{C}$ & $\mathrm{C}$ & $=$ & $\mathrm{A}$ & $\mathrm{C}$ & $\wedge$ & $\mathrm{D}$ & $\mathrm{D}$ & $=$ & $\mathrm{B}$ & $\mathrm{D}$ & $\wedge$ \\
\hline Sujeto 2 & $\mathrm{D}$ & $\mathrm{D}$ & $=$ & $\mathrm{D}$ & $\mathrm{D}$ & $=$ & $\mathrm{B}$ & $\mathrm{B}$ & $=$ & $\mathrm{B}$ & $\mathrm{D}$ & $\wedge$ \\
\hline Sujeto 3 & $\mathrm{A}$ & $\mathrm{B}$ & $\wedge$ & $\mathrm{A}$ & $\mathrm{B}$ & $\wedge$ & $\mathrm{D}$ & $\mathrm{E}$ & $\wedge$ & $\mathrm{D}$ & $\mathrm{D}$ & $=$ \\
\hline Sujeto 4 & $\mathrm{A}$ & $\mathrm{B}$ & $\wedge$ & $\mathrm{A}$ & $\mathrm{E}$ & $\wedge$ & $\mathrm{A}$ & $\mathrm{B}$ & $\wedge$ & $\mathrm{A}$ & $\mathrm{C}$ & $\wedge$ \\
\hline
\end{tabular}




\begin{tabular}{|c|c|c|c|c|c|c|c|c|c|c|c|c|}
\hline Sujeto 5 & $\mathrm{C}$ & $\mathrm{C}$ & $=$ & $\mathrm{E}$ & $\mathrm{E}$ & $=$ & $\mathrm{C}$ & $\mathrm{D}$ & $\wedge$ & $\mathrm{B}$ & $\mathrm{C}$ & $\wedge$ \\
\hline Sujeto 6 & $\mathrm{D}$ & $\mathrm{D}$ & $=$ & $\mathrm{B}$ & $\mathrm{D}$ & $\wedge$ & $\mathrm{B}$ & $\mathrm{B}$ & $=$ & $\mathrm{B}$ & $\mathrm{D}$ & $\wedge$ \\
\hline Sujeto 7 & $\mathrm{B}$ & $\mathrm{D}$ & $\wedge$ & $\mathrm{A}$ & $\mathrm{B}$ & $\wedge$ & $\mathrm{C}$ & $\mathrm{C}$ & $=$ & $\mathrm{D}$ & $\mathrm{D}$ & $=$ \\
\hline Sujeto 8 & $\mathrm{C}$ & $\mathrm{D}$ & $\wedge$ & $\mathrm{A}$ & $\mathrm{D}$ & $\wedge$ & $\mathrm{E}$ & $\mathrm{E}$ & $=$ & $\mathrm{D}$ & $\mathrm{D}$ & $=$ \\
\hline Sujeto 9 & $\mathrm{A}$ & $\mathrm{C}$ & $\wedge$ & $\mathrm{A}$ & $\mathrm{D}$ & $\wedge$ & $\mathrm{C}$ & $\mathrm{C}$ & $=$ & $\mathrm{B}$ & $\mathrm{C}$ & $\wedge$ \\
\hline $\begin{array}{c}\text { Sujeto } \\
10\end{array}$ & $\mathrm{D}$ & $\mathrm{D}$ & $=$ & $\mathrm{A}$ & $\mathrm{E}$ & $\wedge$ & $\mathrm{B}$ & $\mathrm{D}$ & $\wedge$ & $\mathrm{A}$ & $\mathrm{D}$ & $\wedge$ \\
\hline
\end{tabular}

\section{Evaluación del CIMA}

Como se ha reseñado al final del apartado dedicado a la aplicación del CIMA, el trabajo realizado a partir de los cuestionarios ha sido fundamental para poder desarrollar estas sesiones. En primer lugar para ver la percepción que cada alumno tiene del bloque teórico-práctico y poder moldearlo para hacerlo más efectivo. Sin pretender que el alumno tenga que resolver problemas ajenos a lo que es la práctica de la asignatura. Ese es el aspecto que mejor ha permitido hacer las clases más acotadas y no apartarlas del problema principal. De cara al futuro sería interesante plantear una manera de impartir la teoría de manera más orgánica. En vez de basarse en el modelo de explicar los conceptos teóricos tal cual, utilizando algún ejemplo, o directamente con supuestos que den lugar a conceptos teóricos que surjan de las dudas de los alumnos.

A modo de reflexión final se pueden plantear unos principios didácticos que se pueden dividir en dos grandes bloques: el primero, preparar la clase mucho más allá de la mera impartición de los contenidos teóricos; y el segundo, llevar todo el contenido posible al apartado práctico para que el alumno pueda resolver los problemas a partir de la generación de ideas propias. Dentro del primer grupo de principios, estarían: desarrollar una metodología a través del análisis de la práctica docente, crear un modelo metodológico que se adapte al máximo tanto al estudiante como al contenido que se tiene que impartir, 
desarrollar como podemos impartir esos contenidos a través de la creación de actividades específicas y planificar sobre el papel la organización de las clases a través de la formulación de problemas. Dentro del segundo grupo podemos incluir los siguientes principios: conocer la realidad conceptual del alumno a través del desarrollo de cuestionarios, analizar y categorizar las respuestas de los alumnos permitiéndonos apreciar los obstáculos y buscar elementos que nos ayuden en el diseño de las actividades que sirvan a superar dichos obstáculos.

Jornadas de Formación e Innovación Docente del Profesorado | № 2 (2019) Esta obra se distribuye con la licencia Creative Commons 


\section{Referencias bibliográficas}

Bain, K. (2005). Lo que hacen los mejores profesores universitarios. Valencia, España: PUV.

Dolmans, D., De Grave, W., Wolf Agen, I., \& Van der Vleuten, C. (2005). Problem-based learning: future challenges for educational practice and research. Medical Education, 39(7), 732-741.

Finkel, D. (2008). Dar clase con la boca cerrada. Valencia, España: Universitat de València, Servei de Publicacions. Rivero, A. y Porlán, R. (2017). La evaluación en la enseñanza universitaria. En R. Porlán (Coord.), Enseñanza universitaria. Cómo mejorarla (73-91). Madrid, España: Morata.

Jornadas de Formación e Innovación Docente del Profesorado | № 2 (2019) Esta obra se distribuye con la licencia Creative Commons Reconocimiento-NoComercial-SinObraDerivada 4.0 Internacional (CC BY-NC-ND 4.0.) 